\title{
Hopf bifurcation in an open monetary economic system: Taylor versus inflation targeting rules
}

\begin{abstract}
The main aim of the present work is to detect the Hopf bifurcation in policy relevant economic dynamical system. The study employs two deferent forms of monetary policy rules namely: Taylor rule and inflation targeting rule. The results show that there exists Hopf bifurcation between policy relevant variables in both types of rules in our open economic system.
\end{abstract}

Keyword: Hopf bifurcation; Economic system; Policy; Taylor rule; Inflation targeting rule 